\title{
Estudo In Vitro da limpeza de canais simulados (Wave One Gold x W-File)
}

\author{
In Vitro Study of simulated canal cleaning (Wave One Gold x W-File) \\ Estudio In Vitro de limpieza de canales simulados (Wave One Gold x W-File)
}

Recebido: 12/10/2021 | Revisado: 18/10/2021 | Aceito: 18/10/2021 | Publicado: 21/10/2021

\author{
Marcely Reis da Silva \\ ORCID: https://orcid.org/0000-0003-3807-4286 \\ Faculdade de Ilhéus, Brasil \\ E-mail: marcelyrs@outlook.com \\ Adolfo de Matos de Carvalho \\ ORCID: https://orcid.org/0000-0002-4421-660X \\ Faculdade de Ilhéus, Brasil \\ E-mail: adolfodecarvalho@outlook.com \\ Matheus Almeida Rodrigues \\ ORCID: https://orcid.org/0000-0001-9262-3704 \\ Faculdade de Ilhéus, Brasil \\ E-mail: mathsalmeidaaa@gmail.com \\ Edeilton Santana de Oliveira Júnior e Bezerra \\ ORCID: https://orcid.org/0000-0001-6766-8855 \\ Faculdade de Ilhéus, Brasil \\ E-mail: edeiltonjunior@hotmail.com \\ Caio Cesar Souza \\ ORCID: https://orcid.org/0000-0002-6445-5157 \\ ENDOBAHIA Cursos, Brasil \\ E-mail: caioitaobim@hotmail.com \\ Cesar Augusto Perini Rosas \\ ORCID: https://orcid.org/0000-0002-2234-5531 \\ Universidade Estadual do Norte do Paraná, Brasil \\ E-mail: cesarperini66@hotmail.com \\ Ryhan Menezes Cardoso \\ ORCID: https://orcid.org/0000-0003-3072-5347 \\ Instituto Odontológico das Américas, Brasil \\ E-mail:ryhann@ @otmail.com \\ Ana Grasiela da Silva Limoeiro \\ ORCID: https://orcid.org/0000-0003-4633-720X \\ Faculdade de Ilhéus, Brasil \\ E-mail: grasielalimoeiro@gmail.com
}

\begin{abstract}
Resumo
Objetivo: A limpeza e modelagem do canal radicular são etapas importantes do tratamento endodôntico, e pode influenciar no sucesso final do tratamento. O objetivo deste estudo foi comparar a modelagem dos instrumentos reciprocantes Wave One Gold (WOG - Dentisply, Maillefer, Baillagues, Suiça) e W-File (WF - TDK, Shenzlen superfine Technology Co, China) em canais radiculares simulados. Materiais e Métodos: foram utilizados 20 blocos de acrílico, divididos em dois grupos $(\mathrm{n}=10)$ : grupo 1 - WOG; grupo 2 - WF. Antes do preparo do canal radicular, a patência dos canais foi verificada com uma lima K \#10. Imagens fotográficas do pré e pós-operatório foram obtidas para posterior sobreposição e análise em software de imagem (Adobe Photoshop CS5.1). A análise estatística foi feita utilizando teste $\mathrm{T}$ e o teste Mann Whitney. Resultados: mais resina foi removida do lado externo da curva pelo instrumento Wave One Gold em todos os pontos examinados ( $p<0.05)$. Em relação ao transporte do canal, houve diferenças significativas no orifício do canal $(\mathrm{p}<0,05)$. O instrumento Wave One Gold apresentou preparos mais largos. Concluiu-se que tanto o instrumento Wave One Gold, tanto W-File são seguros para uso em canais curvos, promovendo boa limpeza e modelagem, sendo que o WF obteve preparos mais conservadores.
\end{abstract}

Palavras-chave: Canais simulados; Endodontia; Instrumentação; Instrumentos reciprocantes; Wave One Gold; WFile.

\begin{abstract}
Objective: Cleaning and shaping of the root canal are important steps in endodontic treatment and may influence the final success of the treatment. The aim of this study was to compare the modeling of the reciprocal instruments Wave One Gold (WOG - Dentisply, Maillefer, Baillagues, Switzerland) and W-File (WF - Shenzlen superfine Technology Co, China) in simulated root canals. Materials and methods: 20 acrylic blocks were used, divided into two groups $(n=10)$ : group 1 - WOG; group 2 - WF. Before root canal preparation, the patency of the root canal was checked with
\end{abstract}


a \#10 K file. Pre- and postoperative photographic images were created in image processing software (Adobe Photoshop CS5.1) for later overlay and analysis. Statistical analysis was performed using the t-test and Mann Whitney test. Results: With the Wave One Gold instrument, more resin was removed from the outside of the curve at all points examined $(\mathrm{p}<0.05)$. In terms of canal transport, there were significant differences in canal opening $(\mathrm{p}<0.05)$. The Wave One Gold instrument had wider preparations. It was concluded that both the Wave One Gold Instrument and the W-File are safe for use in curved canals and promote good cleaning and shaping, with the WF achieving more conservative preparations.

Keywords: Simulated canals; Endodontics; Instrumentation; Reciprocating instruments; Wave One Gold; W-File.

\section{Resumen}

La limpieza y el modelado del conducto radicular son pasos importantes en el tratamiento de endodoncia y pueden influir en el éxito final del tratamiento. El objetivo de este estudio fue comparar qué instrumento alternativo (Wave One Gold o W-File) promueve la mejor y más adecuada limpieza y modelado de conductos radiculares simulados. Materiales y métodos: se utilizaron 20 bloques acrílicos, divididos en dos grupos de 10 (grupo 1: WOG; grupo 2: WF), que se rellenaron con pintura nankin. Antes de la preparación del conducto radicular, se verificó la permeabilidad del conducto radicular con una lima \# $10 \mathrm{~K}$ y se determinó la longitud de trabajo. Se obtuvieron imágenes fotográficas pre y postoperatorias para su posterior superposición y análisis en software de imágenes (Adobe Photoshop CS5.1). El análisis estadístico se realizó mediante la prueba t y la prueba de Mann Whitney. Resultados: El instrumento Wave One Gold eliminó más resinas del exterior de la curva en todos los puntos examinados ( $\mathrm{p}<0,05)$. En cuanto al transporte del canal, hubo diferencias significativas en el orificio del canal ( $\mathrm{p}$ $<0,05)$. El instrumento Wave One Gold tenía preparaciones más amplias. Se concluyó que tanto el instrumento Wave One Gold como el W-File son seguros para su uso en canales curvos, lo que promueve una buena limpieza y modelado, y el WF obtuvo preparaciones más conservadoras.

Palabras clave: Endodoncia; Instrumentación; Wave One Gold; W-File; Recíprocos; Canales simulados.

\section{Introdução}

A limpeza e modelagem do canal radicular são etapas importantes do tratamento endodôntico, e pode influenciar no sucesso final do tratamento, pois é o meio utilizado para remoção de tecido infectado, obtendo esvaziamento do canal radicular, remoção de smear layer e vai combater os microrganismos presentes e seus subprodutos. Assim como também vai preparar o canal para obturação, dando conicidade adequada (Schilder, 1974).

O movimento reciprocante foi descoberto por Yared em 2008. Reciproc (VDW, Munich, Alemanha) foi a primeira lima de níquel titânio ( $\mathrm{Ni} \mathrm{Ti}$ ) reciprocante introduzida no mercado. O instrumento foi desenvolvido visando a utilização de uma única lima para realização do preparo do canal, obtendo uma instrumentação mais rápida, otimizando o tempo clínico, com menor custo e eliminação de contaminação cruzada por príons (Yared, 2008).

A utilização da técnica reciprocante faz com que haja menor risco de fratura do instrumento causada por tensão de torção, pois o ângulo de rotação é projetado para ser menor do que o limite elástico do instrumento, reduzindo a fadiga do instrumento (Plotino et al. 2012).

O instrumento Wave One Gold (Dentsply Maillefer, Ballaigues, Suiça) é fabricado por um processo térmico próprio da Dentsply, que produz um NiTi superelástico, e este tratamento tem efeito positivo nas propriedades do instrumento, melhorando força e flexibilidade da lima. O instrumento possui secção transversal em paralelogramo (Webber, 2015).

O instrumento W-File (TDK, China) possui características semelhantes ao WOG, como a secção transversal em paralelogramo, além disso, os tamanhos de ponta e conicidade. Estes instrumentos ainda passam por um tratamento térmico que induz uma camada de óxido de titânio na superfície do instrumento, fazendo com que a cor que fique dourada. (Calefi et al., 2020).

Estudos comparando a utilização das limas foram desenvolvidos com o intuito de avaliar a eficiência de cada uma em relação à limpeza, modelagem, fadiga cíclica, à medida que vão sendo produzidos novos instrumentos para que o cirurgião dentista possua parâmetros que o ajude na escolha da lima em cada procedimento (Zanin et al., 2020; Da Silva Limoeiro et al., 2014; Keskin et al., 2018). 
O objetivo deste trabalho foi comparar qual instrumento reciprocante (Wave One Gold ou W-File) promove a melhor e mais adequada limpeza e modelagem dos canais radiculares simulados.

\section{Metodologia}

Vinte blocos de acrílico (IM do Brasil, São Paulo, Brasil) foram divididos em 2 grupos de limas reciprocantes ( $\mathrm{n}=$ 10): grupo WOG - Wave One Gold 25.07 e o grupo WF - W-File 25.07. Os blocos foram preenchidos com tinta nankin preta (Acrilex, China) em seguida, fotografias foram tiradas para registrar as formas originais dos canais. Depois foram envolvidos com papel laminado, para não ocorrer influência do operador durante a instrumentação e depois posicionados em um torno (TORNIN, Belo Horizonte, Brasil) com a curvatura voltada para esquerda.

Durante a instrumentação, todos os canais radiculares simulados foram preparados pelo mesmo operador experiente segundo a indicação dos fabricantes. Todos os canais foram preparados com motor endodôntico iRoot Pro (Bassi/ Easy Equipamentos Odontológicos, Belo Horizonte, Brasil), com os seguintes ângulos de rotação $170^{\circ}-50^{\circ}$ graus. Antes do preparo do canal radicular, a patência dos canais foi verificada com uma lima K \#10 (Dentsply Maillefer, Ballaigues, Suiça).

Todos os canais radiculares mediram $17 \mathrm{~mm}$ de comprimento e o comprimento de trabalho (CT) foi estabelecido em $17 \mathrm{~mm}$. Cada lima foi usada com 3 movimentos de bicada e então retirada do canal e limpa com gaze e os canais irrigados com $5 \mathrm{~mL}$ de água destilada, em seringa hipodérmica e agulha Endo-Eze (Ultradent Products, Utah, Estados Unidos) até que não houvesse detritos nos blocos. Quando a lima atingia o CT, era imediatamente removida e o canal irrigado completamente até que os detritos fossem completamente removidos.

Os blocos de acrílico foram colocados na posição fixa previamente estabelecida e uma fotografia digital pósinstrumentação de cada bloco instrumentado foi tirada. As imagens pré e pós-instrumentação correspondentes foram sobrepostas com Adobe Photoshop CS5.1 (Adobe Systems, San Jose, EUA). Os contornos dos canais foram traçados com um software experimental especialmente desenvolvido, com o qual os espaços entre os canais não preparados e preparados puderam ser medidos (Figura 1).

Figura 1 - Sobreposição de imagens pré e pós instrumentação com os pontos de medição (a) orifício do canal, (b) a meio caminho entre o orifício do canal e o início da curva, (c) o início da curva, (d) o ápice da curva, (e) ponto final do canal simulado.

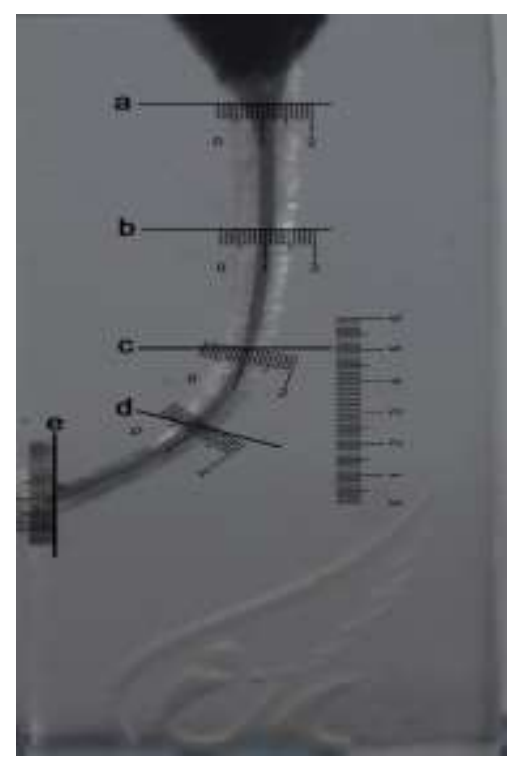

Fonte: Autores. 


\subsection{Avaliação do preparo do canal:}

Cinco pontos foram atribuídos a diferentes níveis de cada imagem do canal. No orifício do canal (a), a meio caminho entre o orifício do canal e o início da curva (b), o início da curva (c), o ápice da curva (d) e o ponto final do canal simulado (e). Os 5 pontos serviram de referência para comparar as imagens pré-instrumentação e pós-instrumentação. (Keskin et al., 2018) (Figura 1).

As imagens de pré-instrumentação e pós-instrumentação foram manipuladas usando o software Adobe Photoshop CS5.1 para que a imagem de pós-instrumentação fosse sobreposta à imagem de pré-instrumentação para auxiliar na avaliação da centralização / transporte do canal. A distância entre o centro de pré-instrumentação traçado e o ponto central pósinstrumentação foram medidos e registrados para cada um dos 5 pontos de referência; esta medição determinou a distância do transporte.

Para medir a capacidade de centralização do canal, a distância do transporte em cada ponto foi medida traçando medidas do centro do canal pré-instrumentado aos pontos de pós instrumentação, onde X1 é a máxima extensão do movimento do instrumento em uma direção (lado interno da curvatura), X2 é o movimento do instrumento na direção oposta e Y é o diâmetro final do preparo do canal. E depois de coletados esses dados foi aplicado na fórmula Total: X1-X2/Y (Lim et al., 2013). (Figura 2).

Figura 2 - X1 representa a máxima extensão do movimento do canal em uma direção (lado Interno da curva.) e X2 é o movimento na direção oposta. Y é o diâmetro do preparo final do canal.

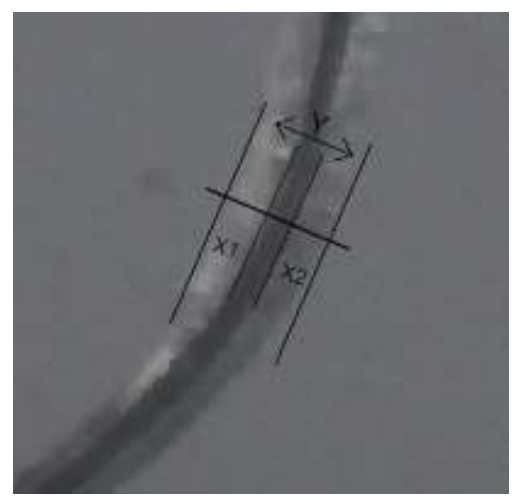

Fonte: Autores.

\subsection{Análise Estatística:}

Foram realizados o teste S Shapiro-Wilk, cuja finalidade é verificar se os dados se apresentam em uma distribuição semelhante a normal $(p>0,05)$ ou não $(p<0,05)$. Nesse caso, verifica-se se essas variáveis estão normalmente distribuídas.

Quando os dados se encontraram normalmente distribuídos, foi lançado mão do teste $\mathrm{T}$ para amostras independentes, quando não se encontravam normalmente distribuídas, foi lançado mão do teste Mann Whitney. Quando $\mathrm{p}<0,05$ há diferenças significativas; quando $\mathrm{p}>0,05$ não há diferenças significativas.

\section{Resultados}

Nenhum instrumento sofreu fratura durante a instrumentação dos canais simulados, e em nenhum preparo ocorreu degrau, perfuração ou algum outro acidente durante o experimento. A largura total dos canais em 5 pontos de medição diferentes após o preparo com W-File e Wave One Gold são apresentados na Tabela 1. 
Tabela 1 - Largura total $(\mathrm{mm})$ do canal em 5 pontos de medição diferentes após a preparação do canal com 2 instrumentos alternativos.

\begin{tabular}{lccc}
\hline Ponto de medição & W-file & Wave One Gold & p-value \\
\hline (a) Orifício do canal & $1,14 \pm 0,16^{\mathrm{a}}$ & $1,43 \pm 0,11^{\mathrm{b}}$ & 0,000 \\
(b) A meio caminho entre o orifício do canal e o & $0,96 \pm 0,14^{\mathrm{a}}$ & $1,23 \pm 0,15^{\mathrm{b}}$ & 0,001 \\
início da curva & $0,86 \pm 0,16^{\mathrm{a}}$ & $1,07 \pm 0,19^{\mathrm{b}}$ & 0,008 \\
(c) Início da curva & $0,62 \pm 0,10^{\mathrm{a}}$ & $0,88 \pm 0,23^{\mathrm{b}}$ & 0,004 \\
(d) Ápice da curva & $0,50 \pm 0,11^{\mathrm{a}}$ & $0,76 \pm 0,24^{\mathrm{b}}$ & 0,004 \\
(e)Ponto final do canal & & \\
\hline
\end{tabular}

Os valores são apresentados como médias \pm desvios padrão. Letras sobrescritas diferentes indicam uma diferença estatisticamente significativa entre os grupos $(\mathrm{p}<0,05)$. Fonte: Autores.

O grupo W-File teve um preparo mais conservador em todos os 5 pontos do canal e o grupo Wave One Gold teve um preparo com maior alargamento do canal.

A Tabela 2 apresenta a quantidade média de resina removida nos 5 pontos pelos instrumentos W-File e Wave One Gold.

Tabela 2 - Medições da largura interna e externa $(\mathrm{mm})$ dos canais em 5 pontos de medição diferentes após a preparação do canal com 2 instrumentos alternativos.

\begin{tabular}{|c|c|c|c|c|c|c|c|c|c|c|}
\hline \multirow[t]{2}{*}{ Instrumento } & \multicolumn{2}{|c|}{$\begin{array}{l}\text { Orifício do canal } \\
\text { (a) }\end{array}$} & \multicolumn{2}{|c|}{$\begin{array}{c}\text { A meio caminho entre } \\
\text { o OC e o início da } \\
\text { curva } \\
\text { (b) }\end{array}$} & \multicolumn{2}{|c|}{$\begin{array}{l}\text { Início da curva } \\
\text { (c) }\end{array}$} & \multicolumn{2}{|c|}{$\begin{array}{c}\text { Ápice da Curva } \\
\text { (d) }\end{array}$} & \multicolumn{2}{|c|}{$\begin{array}{c}\text { Ponto Final da Curva } \\
\text { (e) }\end{array}$} \\
\hline & Interno & Externo & Interno & Externo & Interno & Externo & Interno & Externo & Interno & Externo \\
\hline WF & $0,26 \pm 0,05^{\mathrm{a}}$ & $0,39 \pm 0,15^{\mathrm{a}}$ & $0,23 \pm 0,05^{\mathrm{a}}$ & $0,33 \pm 0,18^{\mathrm{a}}$ & $0,35 \pm 0,08^{\mathrm{a}}$ & $0,14 \pm 0,10^{\mathrm{a}}$ & $0,21 \pm 0,09^{a}$ & $0,1 \pm 0,09^{\mathrm{a}}$ & $0,15 \pm 0,05^{\mathrm{a}}$ & $0,13 \pm 0.09^{a}$ \\
\hline WOG & $0,30 \pm 0,17^{\mathrm{a}}$ & $0,65 \pm 0,19^{b}$ & $0,30 \pm 0,11^{\mathrm{a}}$ & $0,56 \pm 0,21^{\mathrm{b}}$ & $0,51 \pm 0,15^{b}$ & $0,28 \pm 0,19^{b}$ & $0,36 \pm 0,18^{b}$ & $0,26 \pm 0,15^{\mathrm{b}}$ & $0,19 \pm 0,07^{\mathrm{a}}$ & $0,32 \pm 0,18^{b}$ \\
\hline
\end{tabular}

Os valores são apresentados como médias \pm desvios padrão. Letras sobrescritas diferentes indicam uma diferença estatisticamente significativa entre os grupos $(\mathrm{p}<0,05)$. OC - Orifício do canal. Fonte: Autores.

Significativamente mais resina foi removido do lado externo da curva pelo instrumento Wave One Gold em todos os pontos examinados $(\mathrm{p}<0,05)$. Houve diferenças significativas entre as limas Wave One Gold e W-File no lado interno da curva no início da curva (c) e no ápice da curva (d).

A Tabela 3 apresenta os valores médios de transporte independente da direção dos 5 pontos de medição. Nos pontos a meio caminho entre o orifício do canal e o início da curva (b), no início da curva (c), no ápice da curva (d) e no ponto final do canal (e) não houve diferenças significativas entre W-File e Wave One Gold. No orifício do canal (a) houve diferença significativa $(\mathrm{p}<0,05)$. 
Tabela 3 - Distância de transporte do canal $(\mathrm{mm})$ em 5 pontos de medição diferentes após a preparação do canal com 2 instrumentos alternativos.

\begin{tabular}{lccc}
\hline Pontos de medição & W-File & Wave One Gold & p-value \\
\hline (a) Orifício do canal & $-0,10 \pm 0,10^{\mathrm{a}}$ & $-0,24 \pm 0,24^{\mathrm{b}}$ & 0,049 \\
(b) A meio caminho entre o OC e o início da curva & $-0,09 \pm 0,17^{\mathrm{a}}$ & $-0,19 \pm 0,25^{\mathrm{a}}$ & 0,282 \\
(c) Início da curva & $0,26 \pm 0,17^{\mathrm{a}}$ & $0,22 \pm 0,26^{\mathrm{a}}$ & 0,655 \\
(d) Ápice da curva & $0,17 \pm 0,22^{\mathrm{a}}$ & $0,09 \pm 0,31^{\mathrm{a}}$ & 0,504 \\
(e) Ponto final da curva & $0,03 \pm 0,33^{\mathrm{a}}$ & $-0,14 \pm 0,23^{\mathrm{a}}$ & 0,213 \\
\hline
\end{tabular}

Os valores são apresentados como médias \pm desvios padrão. Letras sobrescritas diferentes indicam uma diferença estatisticamente significativa entre os grupos $(\mathrm{p}<0,05)$. OC - Orifício do canal. Fonte: Autores.

\section{Discussão}

Este estudo avaliou a capacidade de limpeza e modelagem em canais curvos simulados com as limas Wave One Gold 25.07 e a lima W-File 25.07. Tendo que levar em conta que dentes naturais apresentam diferentes variações de tamanho, curvatura e forma, o uso dos blocos de acrílico permite padronização desses aspectos citados (Keskin et al., 2018).

Os blocos de acrílico são muito utilizados em pesquisas pois é possível visualizar o que está acontecendo no interior do canal facilitando a comparação de instrumentos e técnicas de preparo (Dummer et al., 1991; Piazza et al., 2021). Contudo, o uso de canais simulados em blocos de acrílico não possui os mesmos resultados quando utilizados dentes naturais, pois a dureza da dentina não é a mesma da resina acrílica (Hülsmann et al., 2003).

Os fabricantes dos dois instrumentos preconizam que as limas sejam de uso único e durante a pesquisa nenhum instrumento sofreu fratura. Isto corrobora com os estudos feitos que avaliam a influência do tratamento térmico das limas que melhoram a resistência a fadiga cíclica e melhora a flexibilidade, reduzindo o risco de fratura (Shen et al., 2013; Ozyurek, 2016; De Menezes et al., 2017; Zanin et al., 2020).

Apesar dos dois instrumentos apresentarem propriedades de secção transversal e conicidades semelhantes, os resultados deste estudo mostrou que a lima Wave One Gold obteve preparos menos conservadores que a W-File, ou seja, os preparos do WOG foram mais largos que os WF. Mais estudos com esses instrumentos devem ser desenvolvidos para que sejam feitas comparações e seja discutida essas diferenças.

O instrumento W-File teve mais capacidade de centralização do canal, e as limas Wave One Gold tiveram um maior transporte para o lado externo da curva em todos os pontos examinados. De acordo com a literatura não foi estudado ainda a capacidade de limpeza comparando as duas limas Wave One Gold e W-File para que fossem feitas comparações diretas com outros trabalhos. Estudos feitos com outros instrumentos já foram feitos em blocos de acrílico para avaliar a capacidade de centralização e transporte do canal, e em canais curvos houve maior transporte para o lado externo da curva com as limas Pro Taper e Hero 642 (Yang et al., 2006).

Um estudo similar em dentes extraídos, utilizando as limas Wave One Gold e ProTaper Next com objetivo de analisar a modelagem dos canais, concluiu que não houve diferenças significativas na centralização de canais entre as limas, mas a Wave One Gold removeu menos dentina (Van Der Vyver et al., 2019). Corroborando com diversos estudos que conclui que movimentos reciprocantes tendem a uma modelagem mais centrada em seu eixo original (Yoo \& Cho, 2012).

Para consolidar ainda mais com o exposto acima, uma pesquisa analisou a influência da trajetória da lima na curvatura do canal e a modificação do eixo após instrumentação de 30 blocos artificiais com Wave One Primary. As imagens foram sobrepostas e foi observado que as modificações do canal parecem ser significativamente reduzidas quando o canal é instrumentado usando a lima de níquel-titânio WaveOne (Berutti et al., 2012). 
A técnica ideal de preparo de um canal é aquela que preserva o máximo da anatomia do canal, significando que a remoção de material é na mesma proporção no lado interior e no exterior dos canais radiculares e minimizando o deslocamento do forame apical (Keskin et al., 2018). O raio da curva do canal influencia no desvio do canal, ou seja, quanto menor o raio de curvatura, mais abruptamente o canal desvia (Pruett et al., 1997).

Comparando a capacidade de transporte das limas, não houve diferenças significativas entre as duas nos pontos examinados, apenas no orifício do canal. Um fator que influencia a modelagem do instrumento é a liga do instrumento. Um estudo anterior utilizando as limas ProTaper Gold (Dentsply Maillefer, Ballaigues, Suíça) comparou-o com os sistemas ProTaper Next (Dentsply Maillefer, Ballaigues, Suiça) e ProTaper Universal (Dentsply Maillefer, Ballaigues, Suiça), e teve como objetivo avalivar a capacidade de modelagem destes instrumentos. Comprovou que as ligas de fio gold comparados com as ligas de m-wire e as ligas de níquel titânio convencional, apresentam menos transporte do canal, resultante do aumento da flexibilidade da liga (Gagliardi et al., 2015).

Um estudo similar foi feito utilizando a lima Endostar E3 Azure ((Poldent, Varsóvia, Polônia), que é um instrumento de níquel-titânio tratados termicamente, em 40 blocos de acrílico. O estudo teve como objetivo avaliar o transporte do canal com a lima sendo operada em rotação continua e reciprocante. Foi realizado a sobreposição de imagens e submetido à análise estatística. Concluiu-se que O Endostar E3 Azure em movimento reciprocante respeitou a curvatura original do canal melhor do que em rotação contínua em todos os níveis, e significativamente obteve menos transporte do canal em $5 \mathrm{~mm}(\mathrm{p}=0,049$, p <0,001) e 7 mm ( $<<0,001, p<0,001$ ) para 20/06 e $25 / 06$ ) (Rebeiz et al., 2021). Comparando com o presente estudo, os instrumentos WOG e WF não demonstraram diferenças significativas no transporte do canal em 4 pontos examinados, tendo diferenças apenas no orifício do canal $(\mathrm{p}<0,05)$, corroborando que as limas tratadas termicamente possuem bons resultados quando operadas em movimentos reciprocantes em relação a outros instrumentos.

\section{Conclusão}

Dentro das limitações deste estudo, ambos os instrumentos, tanto W-File quanto Wave One Gold mostraram-se seguros para uso em canais curvos. O instrumento Wave One Gold obteve preparos mais largos, e a W-File obteve preparos mais conservadores. Não houve diferenças significativas entre os instrumentos em relação ao transporte do canal, mas o instrumento W-File foi o que apresentou maior capacidade de centralização. Contudo, são necessários mais estudos com esses instrumentos para que sejam feitas mais comparações diretas.

\section{Agradecimentos}

Agradeço primeiramente à Deus. Agradeço à minha orientadora Ana Grasiela da Silva Limoeiro pelo incentivo à pesquisa, e todos os autores que fizeram parte deste aprendizado. Agradeço também à minha família por todo apoio.

\section{Referências}

Berutti, E., Paolino, D. S., Chiandussi, G., Alovisi, M., Cantatore, G., Castellucci, A., \& Pasqualini, D. (2012). Root canal anatomy preservation of WaveOne reciprocating files with or without glide path. Journal of endodontics, 38(1), 101-104.

Calefi, P. H. S., Osaki, R. B., Dal Evedove, N. F., Cruz, V. M., de Andrade, F. B. \& Alcalde, M. P. (2020). Cyclic and torcional fatigue resistance of W File and X1 Blue file reciprocating instruments. Dental Press Endodontics. 10(2), 60-66.

Dummer, P. M. H., Alodeh, M. H. A., \& Al-Omari, M. A. O. (1991). A method for the construction of simulated root canals in clear resin blocks. International endodontic Journal, 24(2), 63-66.

Gagliardi, J., Versiani, M. A., de Sousa-Neto, M. D., Plazas-Garzon, A., \& Basrani, B. (2015). Evaluation of the shaping characteristics of ProTaper Gold, ProTaper NEXT, and ProTaper Universal in curved canals. Journal of endodontics, 41(10), 1718-1724.

Hülsmann, M., Gressmann, G., \& Schäfers, F. (2003). A comparative study of root canal preparation using FlexMaster and HERO 642 rotary Ni-Ti instruments. International Endodontic Journal, 36(5), 358-366. 
Keskin, C., Demiral, M., \& Sariyllmaz, E. (2018). Comparison of the shaping ability of novel thermally treated reciprocating instruments. Restorative dentistry \& endodontics, $43(2)$

Da Silva Limoeiro, A. G., Henrique Braitt, A., Rofrigues, E. A., Rabelo bRAItt, G., \& da Silveira bueno, C. E. (2014). Evaluation of the cleaning ability of two rotatory nickel-titanium systems: Protaper universal and heat-treated ProDesign s. Dental Press Endodontics. 34-39.

Lim, Y. J., Park, S. J., Kim, H. C., \& Min, K. S. (2013). Comparison of the centering ability of Wave. One and Reciproc nickel-titanium instruments in simulated curved canals. Restorative dentistry \& endodontics, 38(1), 21-25.

De Menezes, S. E. A. C., Batista, S. M., Lira, J. O. P., \& de Melo Monteiro, G. Q. (2017). Cyclic fatigue resistance of WaveOne Gold, ProDesign R and ProDesign Logic files in curved canals in vitro. Iranian endodontic journal, 12(4), 468.

Özyürek, T. (2016). Cyclic fatigue resistance of Reciproc, WaveOne, and WaveOne Gold nickel-titanium instruments. Journal of endodontics, 42(10), 15361539

Piazza, G. C., Capello, P. A., Ferreira. R., Blatt, J. A., de Araújo. S. M., \& Almeida, C. N. (2021). Avaliação do número de canais modelados com limas ProDesing M até sua fratura, simulado em blocos de resina acrílica. Revista Orbis Science. 1(1), 46-56.

Plotino, G., Grande, N. M., Testarelli, L., \& Gambarini, G. (2012). Cyclic fatigue of Reciproc and WaveOne reciprocating instruments. International Endodontic Journal, 45(7), 614-618.

Pruett, J. P., Clement, D. J., \& Carnes Jr, D. L. (1997). Cyclic fatigue testing of nickel-titanium endodontic instruments. Journal of endodontics, 23(2), 77-85.

Rebeiz, J., El Hachem, C., El Osta, N., Habib, M., Rebeiz, T., Zogheib, C., \& Kaloustian, M. K. (2021). Shaping ability of a new heat-treated NiTi system in continuous rotation or reciprocation in artificial curved canals. Odontology, 1-10.

Schilder, H. (1974). Cleaning and shaping the root canal. Dent Clin North Am, 18, 269-296.

Shen, Y., Zhou, H. M., Zheng, Y. F., Peng, B., \& Haapasalo, M. (2013). Current challenges and concepts of the thermomechanical treatment of nickeltitanium instruments. Journal of endodontics, 39(2), 163-172.

Van Der Vyver, P. J., Paleker, F., Vorster, M., \& Francois, A. (2019). Root canal shaping using nickel titanium, M-wire, and gold wire: A micro-computed tomographic comparative study of one shape, ProTaper next, and WaveOne gold instruments in maxillary first molars. Journal of endodontics, $45(1), 62-67$.

Webber, J. (2015). Shaping canals with confidence: WaveOne GOLD single-file reciprocating system. Roots, 1(3), 34-40.

Yang, G. B., Zhou, X. D., Zhang, H., \& Wu, H. K. (2006). Shaping ability of progressive versus constant taper instruments in simulated root canals. International endodontic journal, 39(10), 791-799.

Yared, G. (2008). Canal preparation using only one Ni-Ti rotary instrument: preliminary observations. International endodontic journal, 41(4), 339-344.

Yoo, Y. S., \& Cho, Y. B. (2012). A comparison of the shaping ability of reciprocating NiTi instruments in simulated curved canals. Restorative dentistry \& endodontics, 37(4), 220-227.

Zanin, E., Langaro, M. C., Pelepenko, L. E., Machado, R. L., Fornari, V. J., \& Hartmann, M. S. M. (2020). Resistência à fratura de instrumentos reciprocantes Wave One Gold e W-File. Full Dentistry In Science. 11(44), 94-98. 